\title{
Regional amyloid accumulation predicts memory decline in initially cognitively unimpaired individuals
}

\author{
Lyduine E. Collij ${ }^{1}$ ｜ Sophie E. Mastenbroek ${ }^{1}$ | Gemma Salvadó2,3 | Alle Meije Wink ${ }^{1}$ \\ Pieter Jelle Visser $^{4}$ | Frederik Barkhof ${ }^{1,5}$ | Bart. N.M. van Berckel ${ }^{1}$ | Isadora Lopes \\ Alves $^{1}$
}

${ }^{1}$ Amsterdam UMC, Department of Radiology and Nuclear Medicine, Vrije Universiteit Amsterdam, Amsterdam, Netherlands

${ }^{2}$ Barcelonaßeta Brain Research Center (BBRC), Pasqual Maragall Foundation,

Barcelona, Spain

${ }^{3}$ IMIM (Hospital del Mar Medical Research Institute), Barcelona, Spain

${ }^{4}$ Amsterdam UMC, Alzheimer Center and department of Neurology, Vrije Universiteit Amsterdam, Amsterdam, Netherlands

${ }^{5}$ Medical Physics and Biomedical Engineering, Centre for Medical Image Computing, UCL, London, UK

\section{Correspondence}

Lyduine E. Collij, Department of Radiology and Nuclear Medicine, VU University Medical Center, De Boelelaan 1117, 1108 HV Amsterdam, The Netherlands.

Email:I.collij@amsterdamumc.nl

\begin{abstract}
Introduction: The value of quantitative longitudinal and regional amyloid beta (A $\beta$ ) measurements in predicting cognitive decline in initially cognitively unimpaired (CU) individuals remains to be determined.

Methods: We selected $133 \mathrm{CU}$ individuals with two or more $\left[{ }^{11} \mathrm{C}\right]$ Pittsburgh compound $\mathrm{B}\left(\left[{ }^{11} \mathrm{C}\right] \mathrm{PiB}\right)$ scans and neuropsychological data from Open Access Series of Imaging Studies (OASIS-3). Baseline and annualized distribution volume ratios were computed for a global composite and four regional clusters. The predictive value of $A \beta$ measurements (baseline, slope, and interaction) on longitudinal cognitive performance was examined.

Results: Global performance could only be predicted by $A \beta$ burden in an early cluster (precuneus, lateral orbitofrontal, and insula) and the precuneus region of interest (ROI) by itself significantly improved the model. Precuneal $A \beta$ burden was also predictive of immediate and delayed episodic memory performance. In $A \beta$ subjects at baseline $(N=93)$, lateral orbitofrontal $A \beta$ burden predicted working and semantic memory performance.

Discussion: Quantifying longitudinal and regional changes in $A \beta$ can improve the prediction of cognitive functioning in initially $\mathrm{CU}$ individuals.

KEYWORDS

Alzheimer's disease, amyloid beta, longitudinal, positron emission tomography, regional
\end{abstract}

\section{1 | INTRODUCTION}

Accumulation of cerebral amyloid beta $(A \beta)$ plaques starts decades prior to Alzheimer's disease (AD)-associated clinical symptom onset, and it is considered a key early event in the cascade leading to $A D$ dementia. ${ }^{1}$ Current secondary prevention trials are aimed at either removing established plaque deposition or halting its accumulation, 2,3 and upcoming trials may focus on subjects with only moderate $A \beta$ burden or even move toward primary prevention strategies. ${ }^{4}$ This shift toward early intervention warrants the need for detecting subtle changes of both $A \beta$ burden as well as cognitive functioning. Therefore, understanding trajectories of $A \beta$ accumulation and its relationship with cognitive decline in initially asymptomatic individuals may be relevant in improving clinical trial design. 
Generally, $A \beta$ burden levels already reach a plateau in preclinical phases. ${ }^{5-8}$ As a consequence, most cognitively impaired patients display established $A \beta$ pathology levels, and the cross-sectional relationship between $A \beta$ and cognition may no longer be evident. ${ }^{5,8,9}$ However, this relationship might be detectable in preclinical stages, where $A \beta$ is still driving the cascade of disease progression. Indeed, a significant association between $A \beta$ burden and cognitive performance in asymptomatic $A \beta$-positive individuals has been shown, ${ }^{10-12}$ but this association was not apparent in the earliest preclinical stages where $A \beta$ plaque deposition is still low. ${ }^{13,14}$ In initially $A \beta$-negative $(A \beta-)$ individuals, $A \beta$ accumulation rather than baseline $A \beta$ burden has been shown to predict a decline in memory, ${ }^{13-15}$ which is considered one of the first domains to deteriorate in AD. ${ }^{16}$ Thus in a preclinical population, $A \beta$ accumulation may be a more meaningful marker of disease stage than the traditional baseline burden, as determined in most studies.

In addition, the value of regional assessments in detecting associations between early $A \beta$ and cognitive performance was recently suggested. ${ }^{13,17}$ More specifically, $A \beta$ accumulation in cortical parietal regions better predicted memory decline compared to global measures of amyloid burden in $A \beta$ - individuals. ${ }^{13}$ This is supported by the finding that the emergence of $A \beta$ plaques seems to follow a spatial-temporal progression, ${ }^{18}$ with a number of studies reporting the precuneal, insular, cingulate, and orbitofrontal cortices as so-called "early accumulating regions." ${ }^{13,19-21}$

The current study aimed to investigate the relationship between amyloid burden and subsequent cognitive decline in an initially cognitively unimpaired (CU) population. More specifically, we assessed whether longitudinal and regional dynamic $\left[{ }^{11} \mathrm{C}\right] \mathrm{Pittsburgh}$ compound $\left.\mathrm{B}\left({ }^{11} \mathrm{C}\right] \mathrm{PiB}\right)$ positron emission tomography (PET) improved the prediction of cognitive change in the Open Access Series of Imaging Studies (OASIS-3).

\section{2 | MATERIALS AND METHODS}

\section{1 | OASIS-3 data set}

Data were obtained from the OASIS-3 data set, a longitudinal neuroimaging, clinical, cognitive, and biomarker data set for normal aging and AD (www.oasis-brains.org). OASIS-3 is a retrospective compilation of data collected across several ongoing projects through the Washington University of Saint Louis Knight Alzheimer's Disease Research Center over the course of 30 years. $^{22}$

\subsection{Participants}

This study included 133 participants selected from the complete OASIS-3 data set $(N=1098)$. Participants were selected when they had (1) two or more $\left[{ }^{11} \mathrm{C}\right] \mathrm{PiB}$ scans at least 1 year apart, (2) at least two neuropsychological assessments, and (3) a Clinical Dementia Rating (CDR) score of 0 . In addition, neuropsychological and PET data were aligned at baseline, with a maximum of 1 year allowed between concomitant assessments (Supplementary Figure 1).

\section{RESEARCH IN CONTEXT}

1. Systematic review: Literature was reviewed using traditional sources (eg, PubMed). Recent work has shown that the extent of regional amyloid beta $(A \beta)$ burden as measured with positron emission tomography (PET) imaging is related to distinct risk of cognitive decline. However, it remains to be determined if continuous quantitative and longitudinal assessments of $A \beta$ burden have added value in predicting cognitive functioning in a preclinical population.

2. Interpretation: Our findings in an initially cognitively unimpaired (CU) population illustrate that longitudinal $A \beta$ PET assessments improved the prediction of changes in both global cognition and memory functioning. In addition, regional $A \beta$ burden in the precuneus and lateral orbitofrontal cortex better predicted cognitive functioning compared to global $A \beta$ measurements.

3. Future directions: These results may be of value to $A D$ prevention trials, where selecting preclinical subjects at high risk of cognitive decline is of great interest. Replication of these results in an independent prospective dataset is warranted.

All participants provided written informed consent and the original study was approved by the Washington University School of Medicine Institutional Review Board.

\section{$2.3 \quad \mid \quad\left[{ }^{11} \mathrm{C}\right] \mathrm{PiB}$ PET imaging}

All participants received an intravenous administration of $6-20 \mathrm{mCi}$ $\left[{ }^{11} \mathrm{C}\right] \mathrm{PiB}$ and were subjected to 60 minutes of dynamic threedimensional (3D) PET imaging. Data were collected on either an ECAT HR+ 962, Biograph 40 PET/CT, or BioGraph mMR PET-MR 3T Siemens PET scanner. Concurring T1-weighted magnetic resonance (MR) scans were obtained using a Siemens 1.5 or 3T MR scanner. ${ }^{22}$ PET images were processed in native space using a local FreeSurferbased processing pipeline (PUP; https://github.com/ysu001/PUP), as described previously. ${ }^{23}$ Reference Logan graphical analysis (RLogan) ${ }^{24}$ with $t^{*}=30$ minutes was used to establish distribution volume ratios (DVRs), using the cerebellar gray matter as reference region.

Global amyloid burden (DVR) was determined based on a mean cortical composite consisting of FreeSurfer-defined frontal, parietal, temporal, and precuneal cortices. ${ }^{23}$ Gaussian mixture modeling (mixtools and AdaptGauss packages from R statistical software program) was used to identify two distributions of global cortical DVR values, and a cut-off for $A \beta$-PET positivity $(A \beta+)$ was determined based on the mean plus 2 standard deviation (SD) from the left Gaussian (ie, "normal") distribution (DVR >1.12). In addition, regional amyloid burden 
was assessed for four clusters of cortical regions, representing different stages in the amyloid accumulation process as described previously by our group. ${ }^{20}$ Specifically, the early accumulating clusters (ie, 1 and 2) consisted of the cingulate cortex, precuneus, insula, and lateral orbitofrontal cortex (LOFC), whereas later accumulating regions (ie, stage 3 and 4) include basal temporal, frontal, and more associative cortices, ending with other temporal and occipital regions.

To account for differences in number of scans and interval between visits, a linear mixed-effects model (LME) with random intercepts and random slopes was used to determine annualized rates of $A \beta$ accumulation (slope).

\subsection{Cognitive assessment}

From a larger cognitive battery, we focused on longitudinal measures of global cognition (Minimal Mental State Examination $[\mathrm{MMSE}]^{25}$ ) and different aspects of memory, including immediate and delayed episodic memory (Logical Memory IA and IIA ${ }^{26}$ respectively), working memory (Digit Span Backward ${ }^{26}$ ), and semantic memory (animal and vegetable Categorical Fluency ${ }^{27}$ ). All memory tests were assessed at the same time point, whereas global cognition was assessed on separate occasions. The available scores were not adjusted for relevant demographic factors. Therefore, all prediction models included age, sex, and level of education as covariates.

\subsection{Statistical analysis}

All statistical analyses were performed in $\mathrm{R}$ version 4.0.2. ${ }^{28}$ Significance was set at $P<.05$, with Bonferroni correction set at $P<.01$ based on the number of cognitive tests as dependent variable (ie, 0.05/5). Differences in sample characteristics and annualized slope between $A \beta-$ and $A \beta+$ subjects were assessed by two-sample $t$-tests and chi-square tests, as appropriate. Non-normality was assessed by a Shapiro-Wilk test, in which case, a nonparametric Mann-Whitney $U$ test was used.

Next, we investigated the relationship between baseline amyloid burden and annualized slope for both the global region of interest (ROI) as well as the amyloid stage clusters. Using the Wilcoxon test, differences in baseline burden and slope between the global ROI and amyloid stages were assessed.

To determine whether longitudinal and regional amyloid burden predicted cognitive decline, accounting for the number of available assessments and follow-up time, we fitted different linear mixed models with subject-specific random intercept and slope. Covariates were age at baseline, sex, years of education, and time between baseline $\left.{ }^{[11} \mathrm{C}\right] \mathrm{PiB}$ and baseline neuropsychological assessments. Thus the following models were applied:

1. Cognition $\sim \mathrm{A} \beta^{*}$ time + covariates

2. Cognition $\sim \mathrm{A} \beta^{*}$ slope $\mathrm{A} \beta^{*}$ time + covariates

Where cognition is performance over time on each of the five preselected cognitive tests (ie, MMSE, Logical Memory IA and IIA, Digit Span
Backward, and Categorical Fluency), $A \beta$ is the continuous $\left[{ }^{11} \mathrm{C}\right] \mathrm{PiB}$ DVR (cross-sectional) and slopeA $\beta$ is the annualized rates of $\left[{ }^{11} \mathrm{C}\right] \mathrm{PiB}$ DVR.

First, we followed a hierarchical approach to assess the relationships between $A \beta$ burden and accumulation and changes in global cognition, using first the global ROI and the four-stage clusters, while the associations at the regional level were only assessed for regions belonging to clusters that were significant in the previous step. Next, we tested the association between $A \beta$ burden and specific memory tests, using the global ROI, four clusters, and individual regions that showed significant predictive value for global cognition. Finally, we investigated the predictive value of regional $A \beta$ burden and memory performance in the $A \beta$ - group. Model preference was based on the Akaike Information Criterion (AIC) ${ }^{29}$ and Bayesian Information Criterion (BIC). ${ }^{30}$ Although both criteria apply a penalty for the number of parameters in the model, the penalty term is larger in BIC than AIC. Delta AIC/BIC was calculated by subtracting the AIC/BIC cross-sectional or global model value from the $\mathrm{AIC} / \mathrm{BIC}$ longitudinal or regional model value, respectively. As lower AIC/BIC values indicate a better model fit, negative delta scores indicate a preference for the longitudinal/regional model.

\section{3 | RESULTS}

\subsection{Demographics of the cohort}

Demographics can be found in Table 1. Subjects underwent 2-13 assessments of global cognition over a period of $7.9 \pm 2.5$ years, and 2-5 neuropsychological assessments over a period of 4.0 \pm 1.9 years. In addition, all subjects underwent 2-5 PET scans over a period of $4.4 \pm 1.9$ years (Supplementary Figure 2). The mean age was $72.2 \pm 5.8$ years at the time of the first neuropsychological assessment, on average $15.7 \pm 2.5$ years of education were completed, the majority of subjects were female (59.4\%), and $32.3 \%$ of subjects were apolipoprotein E gene (APOE) $\varepsilon 4$ carriers.

Regarding cognitive functioning, at baseline, subjects had a mean MMSE score of $28.9 \pm 1.3$ and cognitive performance did not differ between $A \beta^{-}$and $A \beta^{+}$individuals for all cognitive tests, except for the immediate episodic memory task (Logical Memory IA; $A \beta^{-}$: $14.4 \pm 3.8$ $\left.A \beta^{+}: 12.9 \pm 3.9, P=.045\right)$. During the study, seven individuals converted to mild cognitive impairment $(\mathrm{MCl}$ ) and five to dementia (Supplementary Table 1).

\subsection{Amyloid burden and accumulation}

Based on global amyloid burden, $30.1 \%$ of subjects were $A \beta+$ (ie, DVR > 1.12) at the time of the first PET scan. Mean baseline global amyloid burden was 1.14 DVR ( $S D=0.21$, range $=0.92-1.87$ ), and mean change in DVR (ie, slope or $\triangle \mathrm{A} \beta$ ) was $0.01(S D=0.01$, range $=-0.01$ to 0.05$)$. A nonlinear relationship was observed between baseline amyloid burden and slope, with the peak of accumulation around 1.31 
TABLE 1 Sample characteristics

\begin{tabular}{|c|c|c|c|c|}
\hline & Whole cohort & $A \beta$ negative & $A \beta$ positive & $P$ \\
\hline Total, $n$ (\%) & $133(100)$ & $93(69.92)$ & $40(30.08)$ & \\
\hline Follow-up time cognitive sessions (years) & $4.02(1.88)$ & $4.21(1.78)$ & $3.59(2.04)$ & .071 \\
\hline Follow-up time MMSE assessments (y) & $7.89(2.49)$ & $8.19(2.37)$ & $7.17(2.65)$ & .067 \\
\hline $\begin{array}{l}\text { Time between baseline cognitive } \\
\text { assessment and baseline PET scan (days) }\end{array}$ & $164.35(175.68)$ & $173.15(187.06)$ & $143.88(145.94)$ & .910 \\
\hline $\begin{array}{l}\text { Time between baseline MMSE assessment } \\
\text { and baseline PET scan (days) }\end{array}$ & $146.71(111.46)$ & $144.01(111.95)$ & $153(111.46)$ & .631 \\
\hline Education (y) & $15.68(2.52)$ & $15.58(2.47)$ & $15.90(2.64)$ & .446 \\
\hline Converted to dementia, $N(\%)$ & $5(3.76)$ & $3(3.23)$ & $2(5.00)$ & $\mathrm{n} / \mathrm{a}$ \\
\hline \multicolumn{5}{|l|}{ Cognition } \\
\hline Baseline MMSE & $28.92(1.28)$ & $28.95(1.15)$ & $28.85(1.56)$ & .769 \\
\hline Baseline Logical Memory IA & $13.96(3.88)$ & $14.41(3.81)$ & $12.93(3.87)$ & $.045^{*}$ \\
\hline Baseline Logical Memory IIA & $13.16(4.12)$ & $13.58(4.04)$ & $12.18(4.19)$ & .077 \\
\hline Baseline Digit Span Backward & $6.74(2.05)$ & $6.73(2.10)$ & $6.75(1.94)$ & .698 \\
\hline Baseline Categorical Fluency & $17.42(4.13)$ & $17.71(4.10)$ & $16.76(4.16)$ & .231 \\
\hline \multicolumn{5}{|l|}{ PET } \\
\hline
\end{tabular}

A $\beta$-PET positivity was defined as 2 SD above the mean of a normal population based on Gaussian Mixture Modeling (DVR $>1.12$ ). Continuous data are described as mean (SD); categorical data are described as number (percent). Differences in baseline characteristics between the $A \beta$-negative and $A \beta$-positive groups were assessed using two-sample $t$-tests (Baseline Logical Memory IA and IIA and Baseline Categorical Fluency) or non-parametric Mann-Whitney $U$ tests (all other variables) for continuous variables and chi-square tests for categorical variables. * Significant differences between the $A \beta-$ and $A \beta+$ groups. Abbreviations: $A \beta$, amyloid beta; DVR, distribution volume ratio; $\mathrm{MCl}$, mild cognitive impairment; MMSE, Mini-Mental State Exam; PET, positron emission tomography.

global DVR (Figure 1A). This nonlinear relationship was also apparent in cluster 1, 2, and $3 \mathrm{ROIs}$, but less so in cluster 4 , as illustrated by the explained variance associated with a quadratic fit to the data (Figure 1B-E). Compared to the global ROI (baseline: $M=1.14, S D=0.21$; slope: $M=0.011, S D=0.014)$, baseline amyloid burden and amyloid slope were significantly higher in cluster1 (baseline: $M=1.21$, $S D=0.22, P<.001$; Slope: $M=0.012, S D=0.015, P<.001)$ and cluster 2 (baseline: $M=1.19, S D=0.19, P=.002$; slope: $M=0.011$, $S D=0.012, P=.034$ ), but significantly lower in cluster 3 (baseline: $M=1.12, S D=0.01, P=.034$; slope: $M=0.007, S D=0.011, P<.001$ ) and cluster 4 (baseline: $M=1.06, S D=0.14, P<.001$; slope: $M=0.006$, $S D=0.009, P=.003)$.

\subsection{Amyloid burden and change in global cognition}

Full results of the tested models can be found in Table 2. Mixedeffects analyses showed that baseline global amyloid burden did not predict changes in MMSE score. Including global amyloid slope in the model resulted in a significant effect of baseline global amyloid burden $(\beta=-0.32, t=-3.0, P=.003)$ and a marginal effect of the interaction with slope $\left(\mathrm{A} \beta_{\text {global }}{ }^{*} \Delta \mathrm{A} \beta_{\text {global }}: \beta=10.91, t=2.1, P=0.040\right)$ on MMSE. The global amyloid slope by itself was not associated with changes in global cognition ( $\beta=-11.30, t=-1.8, P=0.075)$, and this more complex longitudinal model was not preferred according to AIC or BIC.

At the cluster level, only amyloid burden and accumulation in the cluster 2 ROI could significantly predict changes in MMSE over time, with baseline amyloid being a significant predictor $(\beta=-0.37, t=-3.0$ $\mathrm{P}=.003)$, and its interaction with slope a marginally significant predictor ( $\beta=14.16, t=2.1, P=.038$ ). Cluster 2 slope by itself was not associated with changes in global cognition $(\beta=-15.38, t=-1.8$, $P=0.07)$. This longitudinal model was not preferred compared to the cross-sectional cluster 2 model $(\triangle \mathrm{AIC} / \mathrm{BIC}=6.5 / 21.5$ ) and only slightly preferred compared to the global longitudinal model $(\triangle \mathrm{AIC} / \mathrm{BIC}=$ $-1.9 /-1.9)$. Next, we investigated the predictive value of the cluster 2 
(A)

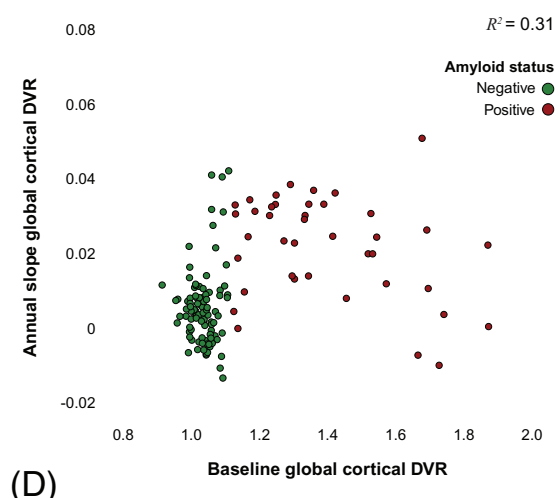

(D)

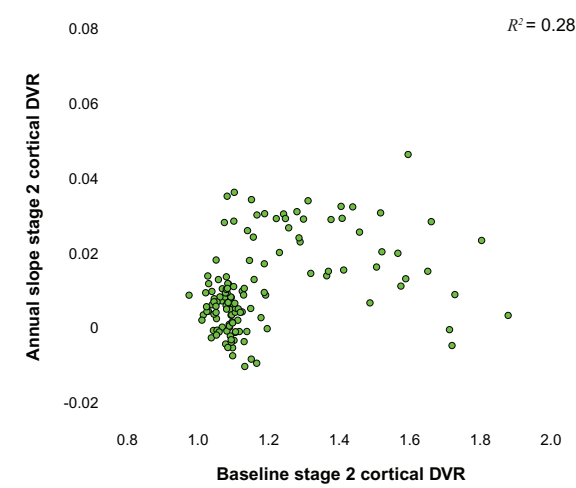

(B)

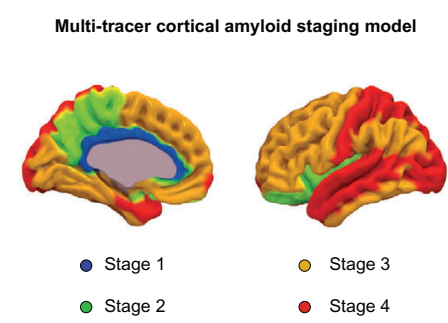

(E)

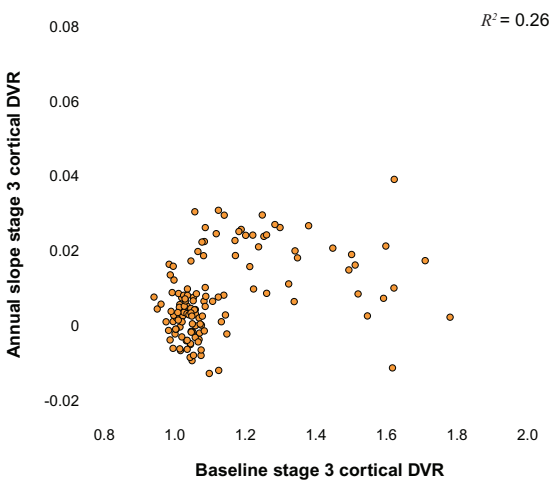

(C)

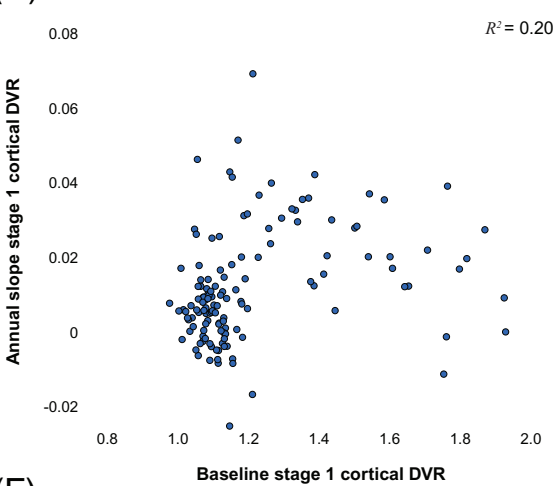

$(\mathrm{F})$

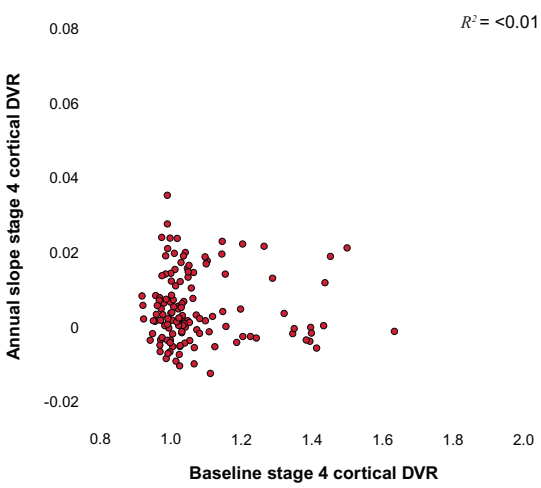

FIGURE 1 Quadratic relationship between baseline DVR and annualized rates of change. Scatterplot of the quadratic relationship between global baseline and annualized rates of change in distribution volume ratios (DVR). For the (A) AD template or global ROI; color-coded for negative (dark green) and positive (dark red) baseline amyloid beta $(A \beta)$ burden. The solid line represents the threshold at 1.12 DVR derived from Gaussian Mixture Modelling and taking the mean plus two SD from the normal population. (B) Representation of the multi-tracer cortical amyloid staging model as published Collij et al., 2020 in Neurology. (C-F) Scatterplots of the quadratic relationship between global baseline and annualized rates of change in DVR for stage 1 (blue), 2 (green), 3 (orange), and 4 (red), respectively

regions on global cognition over time, that is, the precuneus, lateral orbitofrontal (LOFC), and insula.

Precuneal baseline amyloid burden did not significantly predict global cognition over time $(\beta=-0.12, t=-2.1, P=.040)$. When including precuneal $A \beta$ slope and the interaction term $\left(\mathrm{A} \beta_{\text {precuneus }}{ }^{*} \Delta \mathrm{A} \beta_{\text {precuneus }}\right)$ in the model, the predictive power of baseline amyloid burden in this ROI became highly significant $(\beta=$ $-0.29, t=-3.7, P<.001)$, and both precuneus slope $(\beta=-9.71$, $t=-2.2, P=.030)$ and the interaction term $(\beta=8.71, t=2.6$, $P=.011$ ) were marginally predictive of changes in MMSE. In this case, the more complex model including longitudinal PET data was slightly preferred compared to baseline-only predictors based on AIC $(\triangle \mathrm{AIC}=-3.5)$ but not $\mathrm{BIC}(\triangle \mathrm{BIC}=16.5)$. The longitudinal precuneus $\mathrm{ROI}$ model was preferred compared to those using global $(\triangle \mathrm{AIC}=-12.4 / \triangle \mathrm{BIC}=-6.9)$ or stage 2 amyloid $\mathrm{PET}$ measures $(\triangle \mathrm{AIC}=-10.5 / \Delta \mathrm{BIC}=-5.5)$. Finally, amyloid burden measurements in the LOFC and insula were not associated with changes in MMSE.

Figure 2 illustrates the added value of including longitudinal PET data in the predictive models, where a decline in global cognition is apparent only in subjects with both high amyloid burden and low accumulation rates, that is, those in which the $A \beta$ accumulation process has already reached a plateau (red). In contrast, subjects with similarly high baseline amyloid burden but who are still undergoing significant $A \beta$ accumulation (purple) have cognitive decline profiles similar to those who are still $A \beta^{-}$.

\subsection{Amyloid burden and change in memory performance}

Baseline amyloid burden was not predictive of changes in either immediate or delayed episodic memory, independent of the chosen PET ROI (ie, global, cluster, or individual regions). At the regional level, including $A \beta$ slopes in the model was of added value only in the case of the precuneus ROI. Precuneal slope and the interaction between baseline amyloid and accumulation in this region were both marginally predictive of changes in immediate $\left(\beta_{\text {slope }}=-60.95, t=-2.0, P=.053\right.$; $\beta_{\text {interaction }}=53.39, t=2.1 . P=.038$, Figure $3 \mathrm{~A}$ ) and delayed episodic memory $\left(\beta_{\text {slope }}=-65.45, t=-2.2, P=.030 ; \beta_{\text {interaction }}=53.14, t=2.2\right.$, $P=.031$ ), whereas baseline amyloid burden in this ROI was still not associated. These models were not preferred compared to the longitudinal global ROI and cluster $2 \mathrm{ROI}$ model. In the case of semantic and working memory, amyloid burden measures were not predictive 


\begin{tabular}{|c|c|c|c|c|c|c|c|c|c|c|c|}
\hline 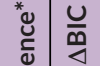 & $\stackrel{\sim}{\tilde{N}}$ & $\stackrel{\sim}{\tilde{N}}$ & $\begin{array}{l}\stackrel{L}{N} \\
\stackrel{\sim}{n}\end{array}$ & 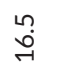 & $\stackrel{\sim}{\underset{\sim}{\sim}}$ & $\stackrel{\text { సై }}{\mathrm{N}}$ & $\stackrel{\stackrel{+}{\sim}}{\sim}$ & 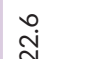 & & 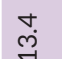 & ָู \\
\hline \begin{tabular}{l|l} 
& $\frac{U}{\Sigma}$ \\
&
\end{tabular} & $\stackrel{n ?}{\sim}$ & $\stackrel{\infty}{0}^{\circ}$ & $\begin{array}{l}\text { In } \\
\text { bु }\end{array}$ & 崖 & $\stackrel{m}{\sim}$ & $\stackrel{+}{\mathrm{N}}$ & ఫू & $\stackrel{\circ}{r}$ & 苂 & $\stackrel{\circ}{\vec{r}}$ & $\stackrel{ユ}{ت}$ \\
\hline $\begin{array}{l}\frac{+}{\underline{u}} \\
\frac{\Delta}{u} \\
\frac{u}{\omega}\end{array}$ & 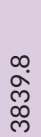 & 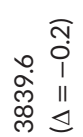 & 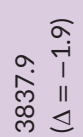 & 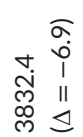 & 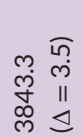 & 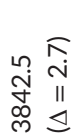 & 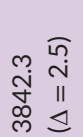 & 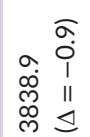 & 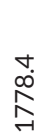 & 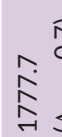 & 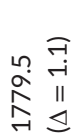 \\
\hline$\frac{\frac{\pi}{0}}{\frac{U}{u}}$ & 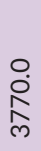 & $\begin{array}{ll} & \widehat{T} \\
\infty & 0 \\
0 & 1 \\
0 & 11 \\
0 & 11 \\
0 & \unlhd\end{array}$ & 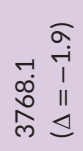 & 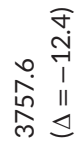 & 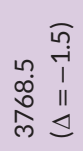 & 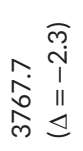 & 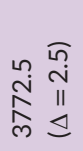 & 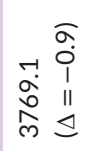 & 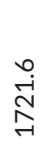 & 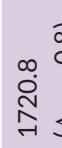 & 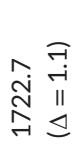 \\
\hline
\end{tabular}

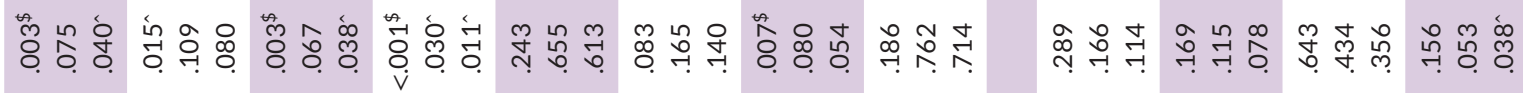

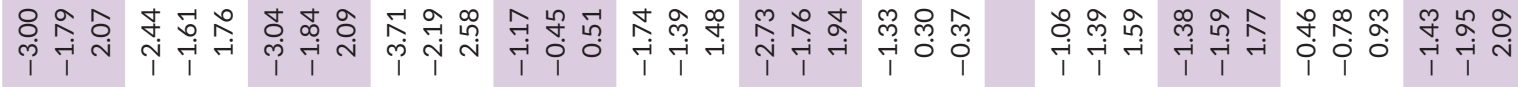

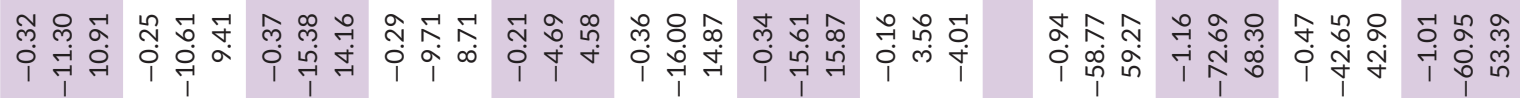

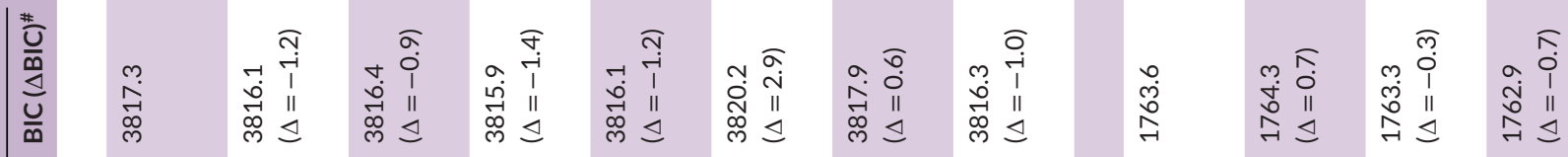

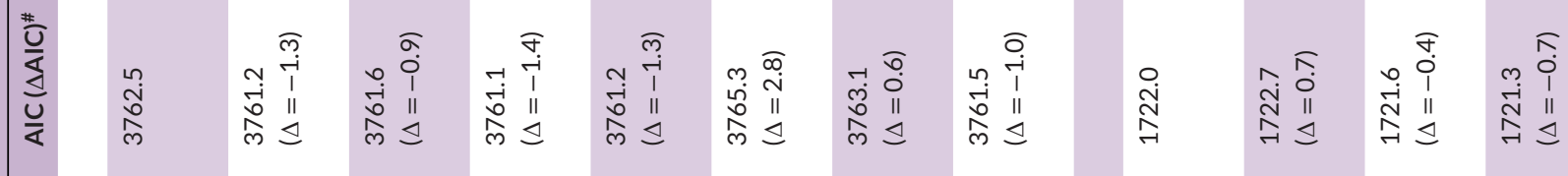

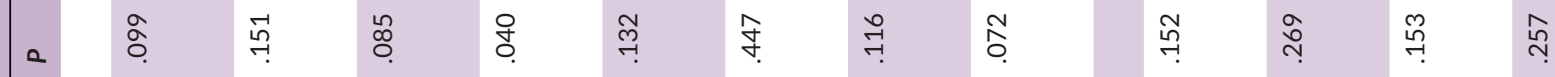

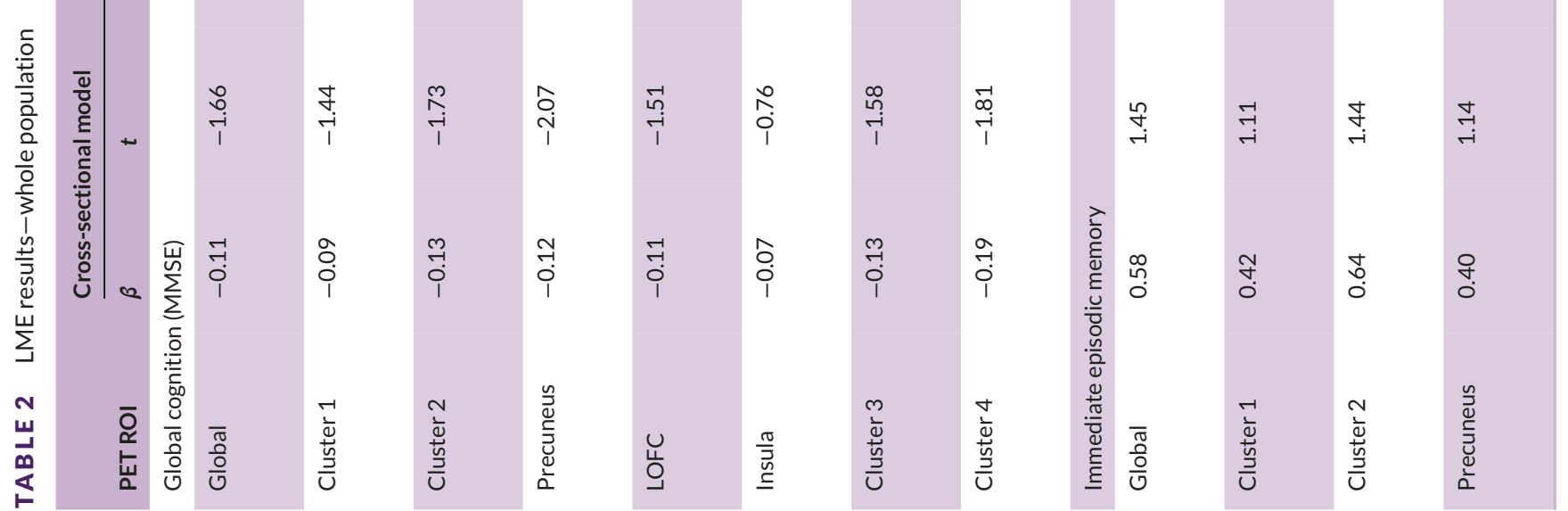




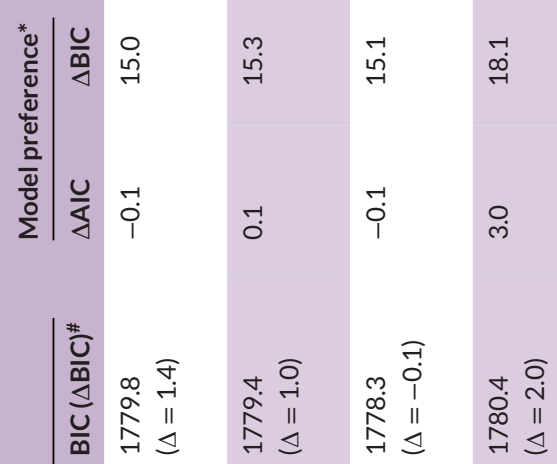

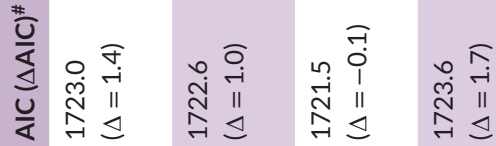

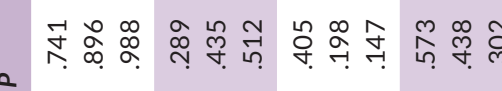

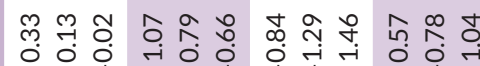

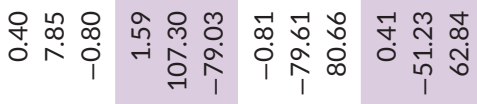

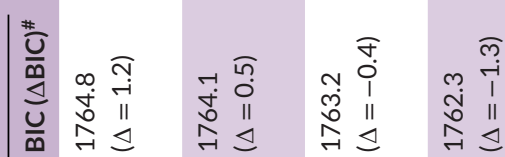

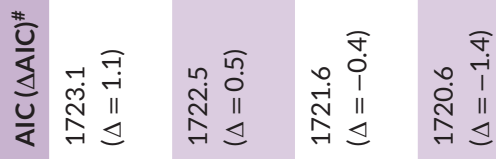

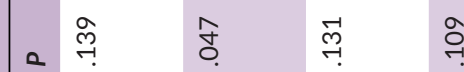

毵

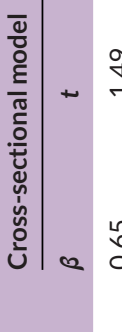

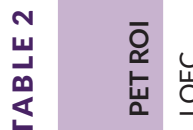

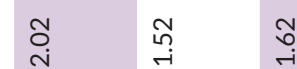

$\Xi$

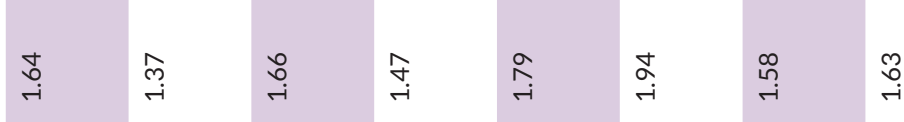

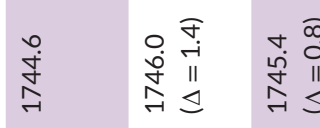

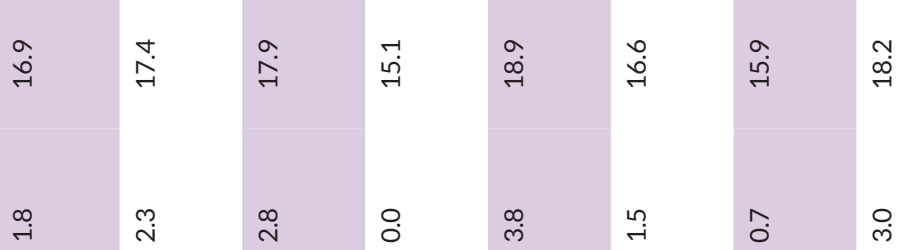

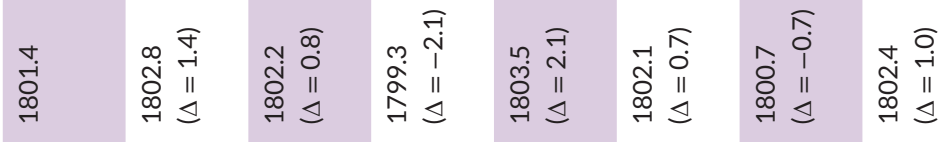

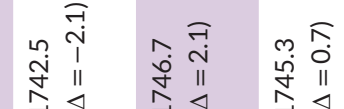

象

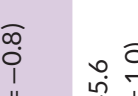

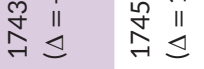

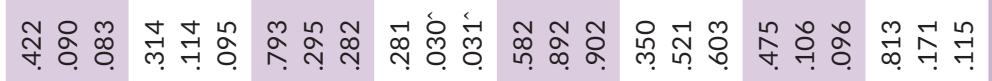

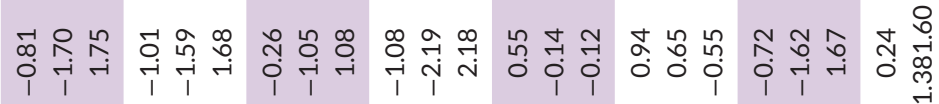

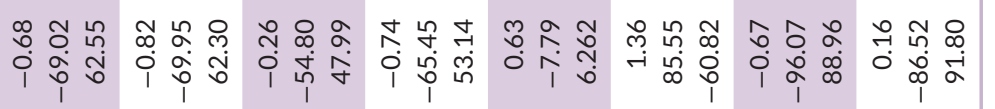

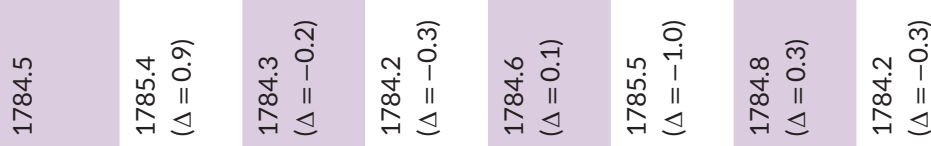

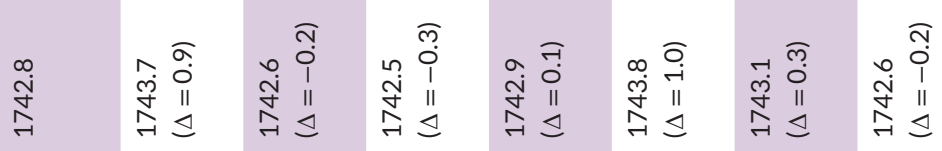

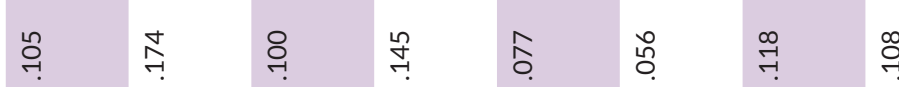

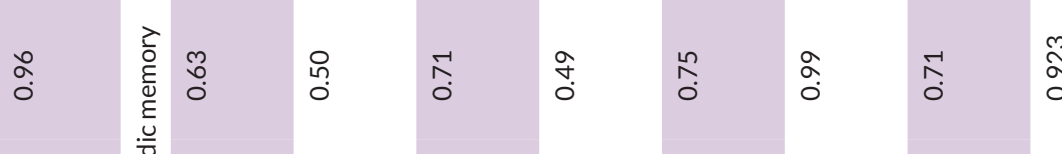

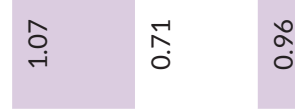

1 旁

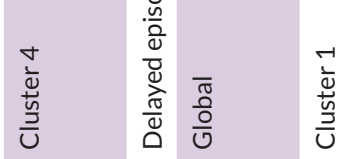

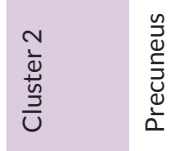

产

衰喜 


\section{(A)}

Cross-sectional Precuneus

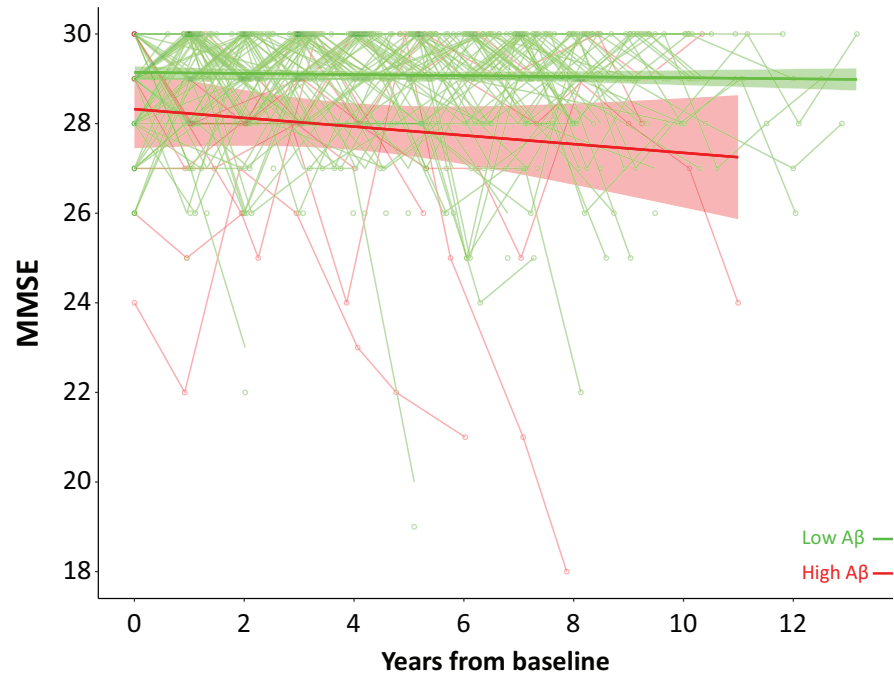

(B)

Longitudinal Precuneus

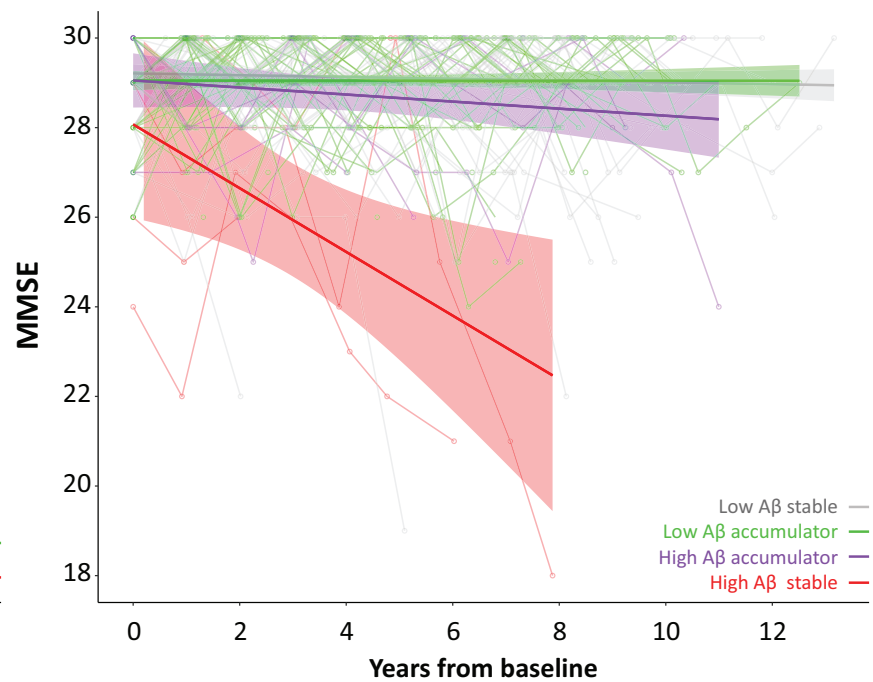

FIGURE 2 FI Relationship between A $\beta$ pathology and decline in global cognition. Illustration of the association between continuous amyloid burden (baseline and longitudinal $\left[{ }^{11} \mathrm{C}\right] \mathrm{PiB}$ PET DVR) and global cognitive functioning (MMSE) over time. The figure reflects the results of the linear mixed effect model including the precuneus ROI; baseline $A \beta$ burden, $A \beta$ slope, and interaction term (A $\beta_{\text {precuneus }}{ }^{*} \Delta A \beta_{\text {precuneus }}$ ). For illustrative purposes, these continuous measures were reduced to categorical groups, with baseline amyloid burden divided into three groups based on k-mean clustering (low [green], gray-zone [not shown], high [red]) and accumulator was defined as percentage change above $0.85 \%$ based on $\left(\left[{ }^{11} \mathrm{C}\right] \mathrm{PiB}\right.$ test-retest data (see Supplemental Figure 3 and Supplemental materials). Colored bands representing the $95 \%$ confidence interval (CI). (A) Changes in MMSE score over time are provided for the subjects with a low A $\beta$ burden (green) and high A $\beta$ burden (red) at baseline. Although the differences in cognitive trajectories between the two groups is apparent, (B) the largest change in MMSE score is observed in those subjects with both high amyloid burden and low accumulation (red), illustrating the value of both cross-sectional and longitudinal amyloid measures to identify subjects a high risk for decline

(A)

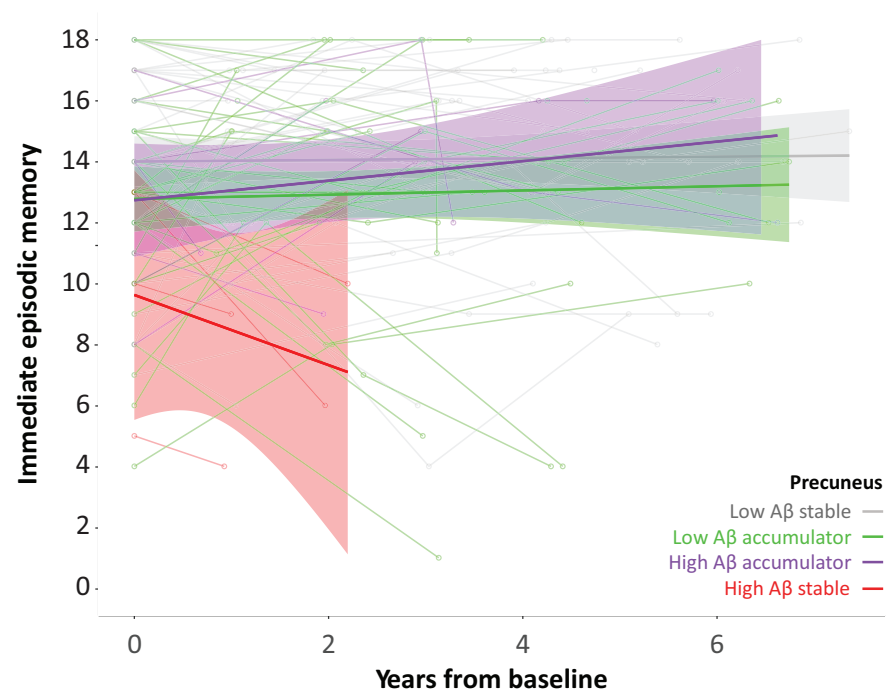

(B)

\section{A $\beta$-negative $(N=93)$}

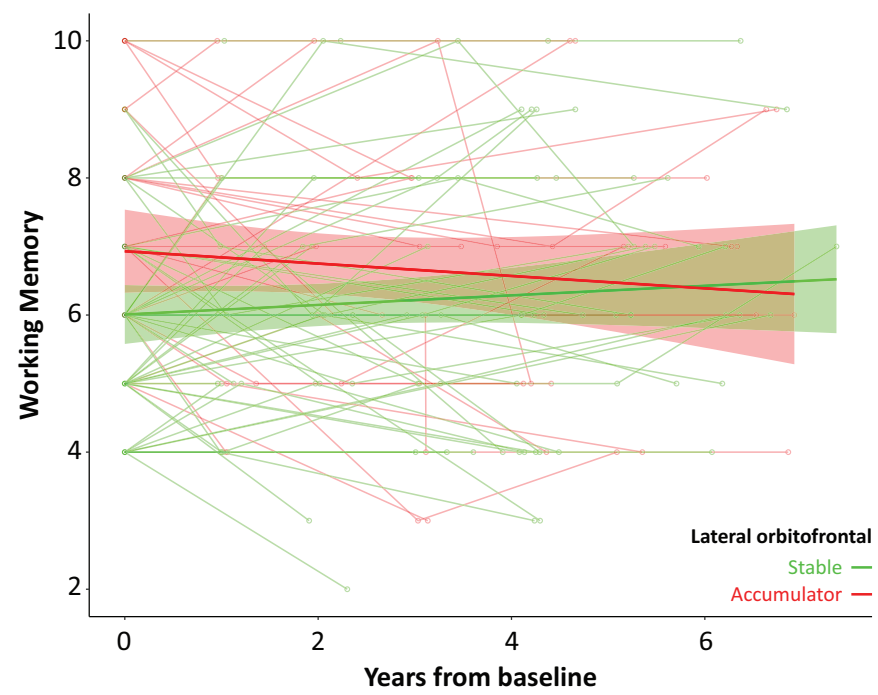

FIGURE 3 F Relationship between A $\beta$ pathology and decline in specific memory tasks. Illustration of the association between continuous amyloid burden (baseline and longitudinal [ $\left.{ }^{11} \mathrm{C}\right]$ PiB PET DVR) and (A) immediate episode memory (Logical Memory IA) performance for the whole population and (B) working memory performance (DIGIB) in the at baseline $A \beta$ - group. The figure reflects the results of the linear mixed-effects model including the (A) precuneus ROI and (B) lateral orbitofrontal (LOFC) ROI; baseline A $\beta$ burden, $A \beta$ slope, and interaction term $\left(A \beta_{R O I}{ }^{*} \Delta A \beta_{R O I}\right)$. For illustrative purposes, the continuous measure slope was reduced to categorical groups, with accumulator defined as percentage change above $0.85 \%$ based on $\left[{ }^{11} \mathrm{C}\right] \mathrm{PiB}$ test-retest data (see Supplemental materials). Colored bands represent $95 \% \mathrm{Cl}$ 
of changes in cognitive performance with any of the ROIs (Supplementary Table 2).

\subsection{Change in cognitive performance restricted to initially $A \beta$-negative subjects}

When restricting the analyses to subjects who were $A \beta-$ at baseline $(N=93)$, the previous associations between precuneal amyloid burden measurements and changes in either global cognition or episodic memory were not found (Supplementary Table 3). Instead, both LOFC slope and the interaction term marginally predicted changes in working $\left(\beta_{\text {slope }}=-171.97 . t=-2.13, P=.038\right.$; $\left.\beta_{\text {interaction }}=-147.62, t=2.02 P=.048\right)$ and significantly predicted semantic memory performance $\left(\beta_{\text {slope }}=-439,43, t=-2.93 P=.004\right.$; $\left.\beta_{\text {interaction }}=393.13, t=2.91, P=.005\right)$. In these models, baseline amyloid burden by itself was not associated with changes in working memory $(P=.578)$ or semantic memory performance $(P=.073)$. Both models were preferred compared to the equivalent global ROI model $\left(\triangle \mathrm{AIC} / \mathrm{BIC}_{\text {working }}=-11.0 /-11.1 ; \quad \Delta \mathrm{AIC} / \mathrm{BIC}_{\text {semantic }}=-8.4 /-\right.$ 8.1) and stage 2 model $\left(\triangle \mathrm{AIC} / \mathrm{BIC}_{\text {working }}=-10.1 / 10.2\right.$; $\left.\triangle \mathrm{AIC} / \mathrm{BIC}_{\text {semantic }}=-9.5 / 9.5\right)$, and slightly to the baseline-only model based on AIC ( $\left.\triangle \mathrm{AIC}_{\text {working }}=-4.1 ; \Delta \mathrm{AIC}_{\text {semantic }}=-1.1\right)$ but not $\mathrm{BIC}\left(\triangle \mathrm{BIC}_{\text {working }}=9.5 ; \mathrm{BIC}_{\text {semantic }}=12.7\right.$, Table 3).

Figure $3 B$ illustrates that in this $A \beta$ - group, accumulation of amyloid pathology (red line) is not associated with a decline in cognitive functioning, but rather a possible lack of learning effect, which is observed for the stable subjects (green line).

\section{4 | DISCUSSION}

The current study aimed to investigate the relationship between amyloid burden and subsequent cognitive decline in an initially cognitively unimpaired (CU) population. We have shown that changes in both global cognition and memory functioning can be predicted by continuous quantitative measures of amyloid burden. Although baseline amyloid burden could only predict changes in global cognition, including $A \beta$ slope improved the models and was necessary to predict changes in performance of specific memory tasks. In addition, $A \beta$ burden in the precuneus better predicted decline in global cognition and episodic memory functioning compared to global $A \beta$ measurements, whereas in initially $A \beta$ - subjects, the key predictive ROI was the LOFC, which was associated with a reduced learning effect in semantic and working memory tests.

In line with previous work, ${ }^{31}$ we observed a non-linear relationship between baseline amyloid burden and rates of change. More specifically, amyloid accumulation rates showed an initial increase in individuals with early amyloid pathology, followed by a maximum, and finally a gradual slowing in individuals with established amyloid pathology. Thus although baseline amyloid burden illustrates the extent of amyloid pathology, rate of change reflects a subject's position in the amyloid accumulation process, enabling the stratification of individu- als according to their placement in the AD disease course and improving risk-profiling efforts. ${ }^{32}$ In individuals classified as $A \beta$ - at baseline, only $A \beta$ slope and its interaction with baseline $A \beta$ burden could predict working and semantic memory performance. This illustrates that longitudinal PET measures are able to identify subjects at the beginning of the amyloid accumulation process, even when they are classified as $A \beta-$ at their baseline visit. Moreover, in this group, the effect of amyloid burden and accumulation on cognitive functioning seems to be related to an absence of learning effect, rather than objective cognitive decline (Figure 3B). These findings are in line with previous work ${ }^{33}$ and further suggest that differences in learning effect could be considered as alternative cognitive end points, in particular, for early secondary or primary prevention trials. It is important to note that our work suggests that longitudinal amyloid PET measurements are also relevant for individuals with established amyloid pathology. As illustrated in Figure 2, subjects with similarly high DVR have distinct risk of cognitive decline depending on whether they are still accumulating amyloid, or whether they have already reached a plateau. In the latter case, subjects are at the end of their the amyloid accumulation process, at which time other process such as tau accumulation may be playing a role and therefore a stronger relationship to cognitive functioning is observed. ${ }^{34}$ This could also explain why the observed association between amyloid burden and MMSE decline was stronger than previously observed in this data set, ${ }^{20}$ where subjects were only staged according to their crosssectional regional amyloid burden and longitudinal information was not accounted for (see Figure 5B in citation ${ }^{20}$ ). Thus information on amyloid rates of change may enable the identification of subjects who not only have amyloid pathology but are also expected to display a significant decline in cognitive performance within the usual (short) time span of a clinical trial. This is crucial information for secondary prevention trials, since these rely on the inclusion of asymptomatic $A \beta+$ subjects and have cognitive performance as the main outcome measure. $^{15,35}$

The value of using regional amyloid burden to predict cognitive decline is also apparent from our work. Previous studies have shown that specific cortical regions display early amyloid pathology and might be more suited to assess amyloid burden in the preclinical stages of AD. ${ }^{19-21}$ In this work, we investigated continuous amyloid burden and amyloid accumulation in clusters of regions described in the multitracer cortical amyloid staging work, representing different stages in the amyloid accumulation process. ${ }^{20}$ We observed that only the stage 2 cluster and its individual cortical regions were predictive of cognitive functioning over time in this early population. More specifically, $A \beta$ burden in the precuneus was a better predictor of cognitive decline in the whole population, whereas the LOFC ROI was associated with a lack in learning effect in the group that was $A \beta-$ at baseline. This differential association between specific ROls, distinct aspects of memory, and disease stage was unexpected, since both amyloid burden in the precuneus and decline in episodic memory performance are consistently implicated in the earliest phases of AD. ${ }^{16,18}$ Nonetheless, the precuneus is suggested to be an important hub in the default mode network (DMN), possibly explaining its particular relationship to episodic memory performance, ${ }^{19,36}$ whereas the association between LOFC 


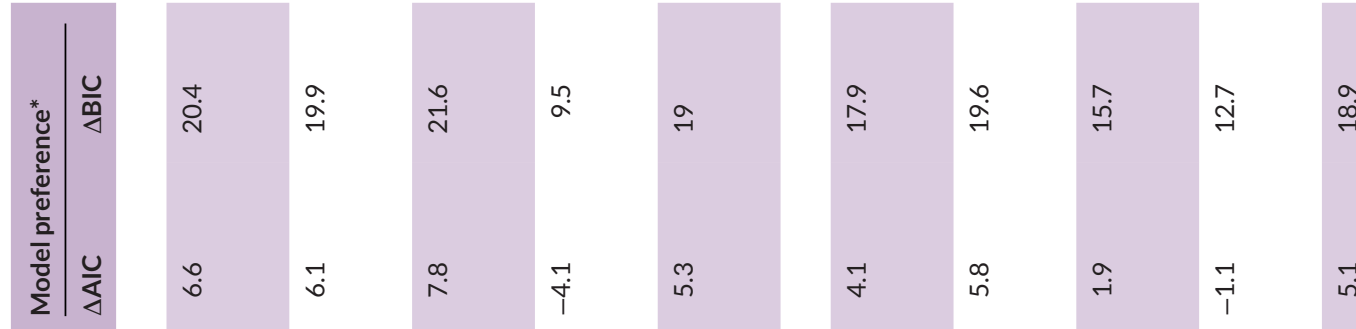

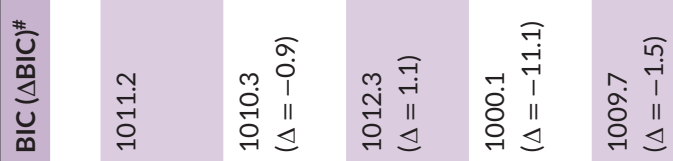

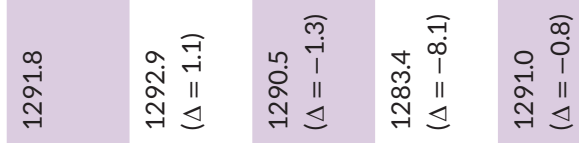

$\frac{\frac{5}{4}}{\frac{2}{4}}$

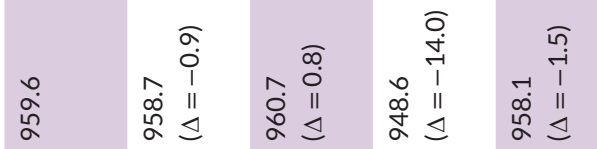

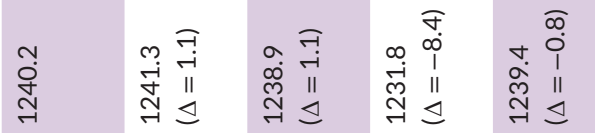

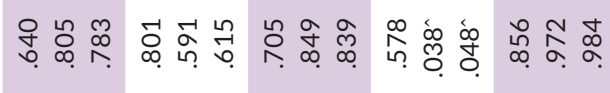

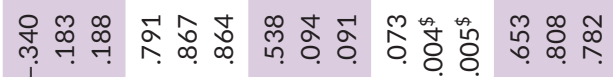

|

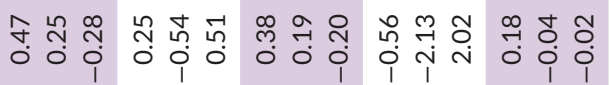

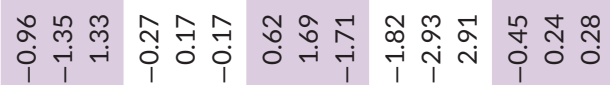

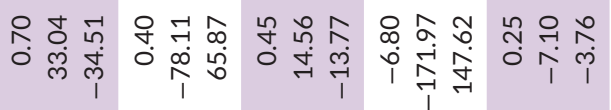

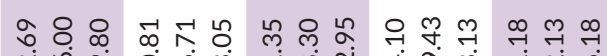

Ү

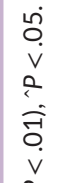

$\frac{u}{0}$

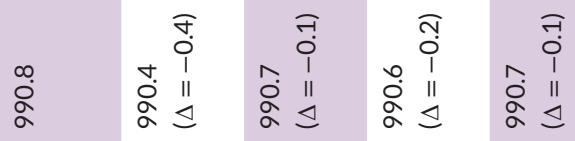

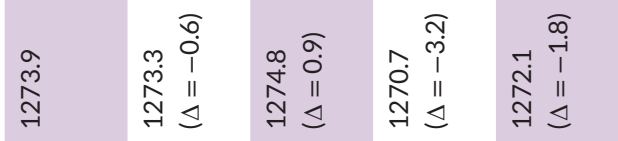

$\frac{⿱ 艹}{\frac{U}{u}}$

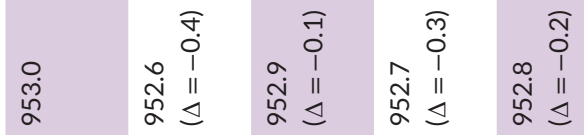

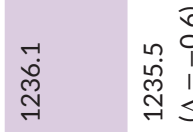

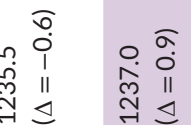

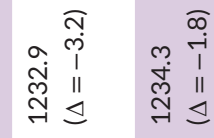

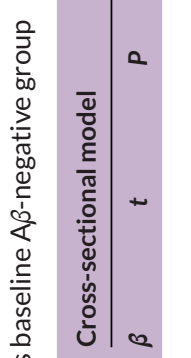

$\stackrel{\infty}{2}$ in

ㄱำ กำ

$\stackrel{m}{\stackrel{m}{0}} \stackrel{\text { ก }}{\circ}$

ปั. กั กั

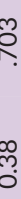

aิ

@i

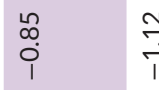

วั

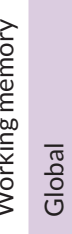
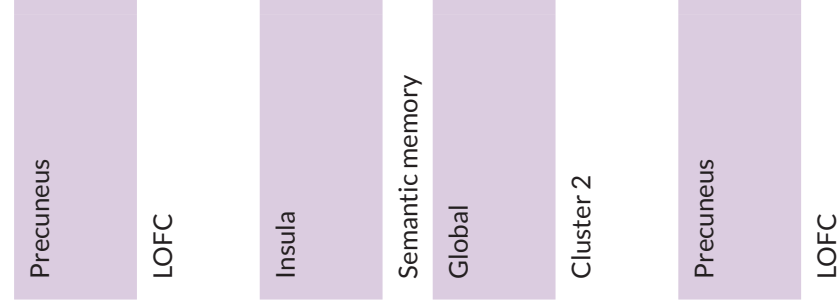

แั

$\stackrel{\frac{\pi}{5}}{\underline{4}}$ 
and working memory could also be explained from a functional perspective, as this region is considered to be essential in this particular memory aspect. ${ }^{37}$ However, it is unclear why each of these ROIs seem to be relevant at different stages of the $A \beta$ accumulation process, especially considering their similar temporal involvement according to amyloid staging work. ${ }^{20,21,38}$

In this work, both AIC and BIC consistently indicated a preference for regional models compared to global or cluster ones. For the group of subjects who were $A \beta$ - at baseline, a consistent AIC/BIC preference for longitudinal regional models was also apparent. However, it is important to note that the longitudinal models were often only slightly preferred compared to the simpler cross-sectional models according to AIC, and not preferred according to BIC. This is probably due to the increased complexity of the models (ie, number of predictors) and the limited sample size of the current work. Thus, validation of the results in a larger sample is of great interest.

Notably, the cingulate cortex (ie, stage 1 cluster) did not show significant associations with cognitive functioning, whereas this region is consistently implicated in early stages of the amyloid accumulation process. ${ }^{19-21,38}$ Of note, different studies point to distinct subregions of the cingulate (anterior, isthmus, posterior) and of those, the posterior cingulate cortex has been most consistently implicated in preclinical ${ }^{13}$ and clinical ${ }^{39}$ stages of AD. However, in this work, these three cingulate sub-regions as defined by the Desikan-Killian (DK) atlas ${ }^{40}$ were clustered for consistency with the multi-tracer staging mode ${ }^{20}$ and in order to reduce technical biases introduced by the quantification of regions with volume close to the PET spatial resolution limit. Yet, our results showed that the cingulate indeed display higher $A \beta$ burden at baseline and amyloid slope compared to a global ROI. However, this increased signal was accompanied by higher variability (Figure 1C), which might have hampered the power in detecting a relationship with cognitive decline. The sources of this variability could be several, such as spill-in from adjacent white matter ${ }^{41}$ or other physiological changes that might not reflect $A D$ processes. Alternatively, the cingulate regions are less implicated in memory performance and might therefore still harbor predictive power for other domains of cognitive functioning.

Some methodological aspects should be considered when interpreting the results of this work. The current study focused only on different aspects of memory, considering our limited sample size and population of interest, for which changes in memory performance are considered to most sensitive measure. ${ }^{16}$ Nonetheless, recent work has reported a decline in both attention and executive functioning, even in CU individuals. ${ }^{42-44}$ Future work should therefore also focus on other cognitive domains associated with early AD. In addition, it would be of interest to investigate the role of cognitive reserve (CR) on the relationship between amyloid burden and cognitive decline. In pre-dementia stages of $A D$, greater $C R$ is associated with attenuated cognitive decline, whereas after the onset of dementia, it seems to be associated with accelerated cognitive decline. ${ }^{45}$ Although studies so far suggest only an effect of CR in the more advanced staged of disease, the role of $C R$ in an early population is still unknown. Third, the cluster- ing of regions was based on previous work ${ }^{20}$ where the OASIS-3 data set was also included. This clustering was data-driven and not based on the relationship with cognitive functioning. Nonetheless, the identification of these regions-of-interest is not fully independent of the currently investigated data set and some circular analysis cannot be excluded. Of note, the selected regions are in line with previous literature in the field of early amyloid burden or amyloid staging, ${ }^{18,19,21}$ and in this work we have specifically focused on assessing the value of continuous and longitudinal quantitative amyloid measures to predict not only changes in global cognition but also specific memory tests. Therefore, replication of these results in an independent prospective data set is important and already planned within the Amyloid Imaging to Prevent Alzheimer's Disease (AMYPAD) consortium. ${ }^{32}$ Fourth, due to the focus on a CU population, the study sample had a relatively limited representation of high $A \beta$ burden levels, and partial volume error (PVE) correction was not performed because considerable atrophy was not expected. In fact, previous work in this data set demonstrated a strong correlation between PVE-corrected and PVE-uncorrected quantitative values, ${ }^{23}$ suggesting that this methodological aspect would likely have minimal effect on our results. In addition, even though we were able to demonstrate subtle changes in the memory domain, longer follow-up is required to confirm whether these accumulating individuals will actually progress to clinical impairment over time. Fifth, it should be noted that visual assessment was not available in this data set and therefore our data-driven cut-off for $A \beta$ positivity based on global DVR values could have resulted in the inclusion of a limited number of subjects with emerging amyloid pathology in the $A \beta$ - subjects sub-analyses. Finally, the RLogan model used to obtain quantitative $A \beta$ measures has been known to underestimate true tracer binding. ${ }^{46}$ In addition, the time interval used in this data set (ie, 30-60 min postinjection [p.i.]) might not be optimal for quantification, considering that the equilibrium of the $\left[{ }^{11} \mathrm{C}\right] \mathrm{PiB}$ radiotracers is around 60 minutes, p.i. and the proposed interval goes until 90 minutes, p.i.. ${ }^{47}$ This could have impacted the strength of the observed relationships with cognitive performance.

\section{5 | CONCLUSION}

The findings in the present study provide further support to the value of both longitudinal and regional assessment of amyloid pathology for the prediction of cognitive changes in initially $\mathrm{CU}$ individuals. These results may be of value to AD prevention trials, where selecting preclinical subjects at high risk of cognitive decline is of great interest. Replication of these results in an independent prospective data set is warranted and already planned within the AMYPAD Prognostic and Natural History Study (PNHS). ${ }^{32}$

\section{ACKNOWLEDGMENTS}

The authors would like to acknowledge the OASIS-3 project. This research would not have been possible without the time and dedication of every participant included in this study. 


\section{FUNDING INFORMATION}

This project has received funding from the Innovative Medicines Initiative 2 Joint Undertaking under grant agreement No. 115952. This Joint Undertaking receives support from the European Union's Horizon 2020 research and innovation program and EFPIA. This communication reflects the views of the authors and neither IMI nor the European Union and EFPIA are liable for any use that may be made of the information contained herein. FB is supported by the NIHR UCLH biomedical research center. FB and AMW are supported by the European Union's Horizon 2020 research and innovation program under grant agreement No. 666992. Data were provided by OASIS-3. Principal Investigators: T. Benzinger, D. Marcus, J. Morris; NIH P50AG00561, P30NS09857781, P01AG026276, P01AG003991, R01AG043434, UL1TR000448, and R01EB009352.

\section{CONFLICTS OF INTEREST}

Lyduine E. Collij, Sophie E. Mastenbroek, Gemma Salvadó, Alle Meije Wink, and Isadora Lopes Alves report no disclosures relevant to this manuscript.

Pieter Jelle Visser has served as member of the advisory board of Roche Diagnostics. Dr Visser received non-financial support from GE Healthcare; research support from Biogen; and grants from BristolMyers Squibb, EU/EFPIA Innovative Medicines Initiative Joint Undertaking, EU Joint Programme-Neurodegenerative Disease Research (JPND and ZonMw).

Frederik Barkhof received payment and honoraria from Bayer Genzyme, Biogen-Idec, TEVA, Merck, Novartis, Roche, IXICO Ltd, GeNeuro, and Apitope Ltd for consulting; payment from the IXICOLtd, and MedScape for educational presentations; and research support via grants from EU/EFPIA Innovative Medicines Initiative Joint Undertaking (AMYPAD consortium), EuroPOND (H2020), UK MS Society, Dutch MS Society, PICTURE (IMDI-NWO), NIHR UCLH Biomedical Research Centre (BRC), and ECTRIMS-MAGNIMS.

Bart N.M. van Berckel received research support from ZON-MW, AVID radiopharmaceuticals, CTMM, and Janssen Pharmaceuticals. $\mathrm{BvB}$ is a trainer for Piramal and GE; he receives no personal honoraria.

\section{DATA AVAILABILITY STATEMENT}

The OASIS-3 data sets hosted by central.xnat.org provide the community with open access to a significant database of neuroimaging and processed imaging data across a broad demographic, cognitive, and genetic spectrum an easily accessible platform for use in neuroimaging, clinical, and cognitive research on normal aging and cognitive decline. All data are available via www.oasis-brains.org.

\section{REFERENCES}

1. Jansen WJ, Ossenkoppele R, Knol DL, et al. Prevalence of cerebral amyloid pathology in persons without dementia: a meta-analysis. JAMA. 2015;313:1924-1938.

2. Huang LK, Chao SP, Hu CJ. Clinical trials of new drugs for Alzheimer disease. J Biomed Sci. 2020;27:18.

3. Sperling R, Mormino E, Johnson K. The evolution of preclinical Alzheimer's disease: implications for prevention trials. Neuron. 2014;84:608-622.
4. Wimo A, Jonsson L, Bond J, Prince M, Winblad B. Alzheimer Disease I. The worldwide economic impact of dementia 2010. Alzheimers Dement. 2013;9:1-11 e3.

5. Engler H, Forsberg A, Almkvist O, et al. Two-year follow-up of amyloid deposition in patients with Alzheimer's disease. Brain. 2006;129:28562866.

6. Hampel H. Amyloid- $\beta$ and cognition in aging and Alzheimer's disease: molecular and neurophysiological mechanisms. J Alzheimers Dis. 2013;33 Suppl 1:S79-86.

7. Jack CR, Jr, Knopman DS, Jagust WJ, et al. Tracking pathophysiological processes in Alzheimer's disease: an updated hypothetical model of dynamic biomarkers. Lancet Neurol. 2013;12:207-216.

8. Jack CR, Jr, Lowe VJ, Weigand SD, et al. Serial PIB and MRI in normal, mild cognitive impairment and Alzheimer's disease: implications for sequence of pathological events in Alzheimer's disease. Brain. 2009;132:1355-1365

9. Arriagada PV, Growdon JH, Hedley-Whyte ET, Hyman BT. Neurofibrillary tangles but not senile plaques parallel duration and severity of Alzheimer's disease. Neurology. 1992;42:631-639.

10. Landau SM, Mintun MA, Joshi AD, et al. Amyloid deposition, hypometabolism, and longitudinal cognitive decline. Ann Neurol. 2012;72:578-586

11. Mormino EC, Betensky RA, Hedden T, et al. Synergistic effect of $\beta$ amyloid and neurodegeneration on cognitive decline in clinically normal individuals. JAMA Neurol. 2014;71:1379-1385.

12. Resnick SM, Sojkova J, Zhou Y, et al. Longitudinal cognitive decline is associated with fibrillar amyloid-beta measured by [11C]PiB. Neurology. 2010;74:807-815.

13. Farrell ME, Chen X, Rundle MM, Chan MY, Wig GS, Park DC. Regional amyloid accumulation and cognitive decline in initially amyloidnegative adults. Neurology. 2018;91:e1809-e21.

14. Landau SM, Horng A, Jagust WJ. Memory decline accompanies subthreshold amyloid accumulation. Neurology. 2018;90:e1452-e60.

15. Insel PS, Donohue MC, Sperling R, Hansson O, Mattsson-Carlgren N The A4 study: $\beta$-amyloid and cognition in 4432 cognitively unimpaired adults. Ann Clin Transl Neurol. 2020;7:776-785.

16. Ritchie K, Ropacki M, Albala B, et al. Recommended cognitive outcomes in preclinical Alzheimer's disease: consensus statement from the European Prevention of Alzheimer's Dementia project. Alzheimers Dement. 2017;13:186-195.

17. Bischof GN, Jacobs HIL. Subthreshold amyloid and its biological and clinical meaning: long way ahead. Neurology. 2019;93:72-79.

18. Fantoni E, Collij L, Lopes Alves I, Buckley C, Farrar G. The SpatialTemporal Ordering of Amyloid Pathology and Opportunities for PET Imaging. J Nucl Med. 2020;61:166-171.

19. Palmqvist S, Schöll M, Strandberg O, et al. Earliest accumulation of $\beta$-amyloid occurs within the default-mode network and concurrently affects brain connectivity. Nat Commun. 2017;8:1214.

20. Collij LE, Heeman F, Salvado G, et al. Multitracer model for staging cortical amyloid deposition using PET imaging. Neurology. 2020;95:e1538-e53.

21. Mattsson N, Palmqvist S, Stomrud E, Vogel J, Hansson O. Staging $\beta$ amyloid pathology with amyloid positron emission tomography. JAMA Neurol. 2019;76:1319-1329.

22. LaMontagne PJ, Benzinger TLS, Morris JC, et al. OASIS-3: longitudinal neuroimaging, clinical, and cognitive dataset for normal aging and Alzheimer disease. medRxiv. 2019. 2019.12.13.19014902.

23. Su Y, D'Angelo GM, Vlassenko AG, et al. Quantitative analysis of PiBPET with FreeSurfer ROIs. PLoS One. 2013;8:e73377.

24. Logan J, Fowler JS, Volkow ND, Wang GJ, Ding YS, Alexoff DL. Distribution volume ratios without blood sampling from graphical analysis of PET data. J Cerebral Blood Flow Metab. 1996;16:834-840.

25. Folstein MF, Folstein SE, McHugh PR. "Mini-mental state". A practica method for grading the cognitive state of patients for the clinician. $J$ Psychiatr Res. 1975;12:189-198. 
26. Elwood RW. The Wechsler Memory Scale-Revised: psychometric characteristics and clinical application. Neuropsychol Rev. 1991;2:179201.

27. Morris JC, Edland S, Clark C, et al. The consortium to establish a registry for Alzheimer's disease (CERAD). Part IV. Rates of cognitive change in the longitudinal assessment of probable Alzheimer's disease. Neurology. 1993;43:2457-2465.

28. Team R. RStudio: integrated development for R. RStudio, Inc, Boston, MA. 2015;42:14. http://www.rstudio.com. Accessed 01, 2020.

29. Akaike H. Factor analysis and AIC. Selected papers of hirotugu akaike. Springer; 1987:371-386.

30. Schwarz G. Estimating the dimension of a model. The Annals of Statistics. 1978;6:461-464.

31. Villemagne VL, Burnham S, Bourgeat $P$, et al. Amyloid beta deposition, neurodegeneration, and cognitive decline in sporadic Alzheimer's disease: a prospective cohort study. Lancet Neurol. 2013;12:357-367.

32. Lopes Alves I, Collij LE, Altomare D, et al. Quantitative amyloid PET in Alzheimer's disease: the AMYPAD prognostic and natural history study. Alzheimers Dement. 2020.

33. Weintraub S, Carrillo MC, Farias ST, et al. Measuring cognition and function in the preclinical stage of Alzheimer's disease. Alzheimers Dement (N Y). 2018;4:64-75.

34. Jack CR Jr, Knopman DS, Jagust WJ, et al. Hypothetical model of dynamic biomarkers of the Alzheimer's pathological cascade. Lancet Neurol. 2010;9:119-128.

35. Sperling RA, Rentz DM, Johnson KA, et al. The A4 study: stopping AD before symptoms begin?. Sci Transl Med. 2014;6:228fs13.

36. Cavanna AE, Trimble MR. The precuneus: a review of its functional anatomy and behavioural correlates. Brain. 2006;129:564-583.

37. Barbey $A K$, Koenigs M, Grafman J. Orbitofrontal contributions to human working memory. Cereb Cortex. 2011;21:789-795.

38. Grothe MJ, Barthel $\mathrm{H}$, Sepulcre J, et al. In vivo staging of regional amyloid deposition. Neurology. 2017;89:2031-2038.

39. Herholz K. Guidance for reading FDG PET scans in dementia patients. Q J Nucl Med Mol Imaging. 2014;58:332-343.

40. Desikan RS, Segonne F, Fischl B, et al. An automated labeling system for subdividing the human cerebral cortex on MRI scans into gyral based regions of interest. Neuroimage. 2006;31:968-980.
41. Smith A, Buckley C, [18F]flutemetamol PET image representation of $\mathrm{Ab}$ pathology; differences between lateral and medial image intensity for equivalent levels of pathology. 10th Human Amyloid Imaging. Miami, FL, USA, 2016.

42. Monsell SE, Mock C, Hassenstab J, et al. Neuropsychological changes in asymptomatic persons with Alzheimer disease neuropathology. Neurology. 2014;83:434-440.

43. Rajan KB, Wilson RS, Weuve J, Barnes LL, Evans DA. Cognitive impairment 18 years before clinical diagnosis of Alzheimer disease dementia. Neurology. 2015;85:898-904.

44. Wilson RS, Leurgans SE, Boyle PA, Bennett DA. Cognitive decline in prodromal Alzheimer disease and mild cognitive impairment. Arch Neurol. 2011;68:351-356.

45. van Loenhoud AC, van der Flier WM, Wink AM, et al. Cognitive reserve and clinical progression in Alzheimer disease: a paradoxical relationship. Neurology. 2019;93:e334-e46.

46. Kimura Y, Naganawa M, Shidahara M, Ikoma Y, Watabe H. PET kinetic analysis -pitfalls and a solution for the Logan plot. Ann Nucl Med. 2007;21:1-8.

47. Price JC, Klunk WE, Lopresti BJ, et al. Kinetic modeling of amyloid binding in humans using PET imaging and Pittsburgh Compound-B. J Cereb Blood Flow Metab. 2005;25:1528-1547.

\section{SUPPORTING INFORMATION}

Additional supporting information may be found online in the Supporting Information section at the end of the article.

How to cite this article: Collij LE, Mastenbroek SE, Salvadó G, et al. Regional amyloid accumulation predicts memory decline in initially cognitively unimpaired individuals. Alzheimer's

DementAlzheimer's Dement. 2021;13:e12216.

https://doi.org/10.1002/dad2.12216 